\title{
Overview of Improved Droop Control Methods of Hybrid AC/DC Microgrid Interlinking Converter
}

\author{
Yajie Guo ${ }^{a}$, Yingjun Guo ${ }^{b}$, Hexu Sun ${ }^{c}$, Jianlin $\mathrm{Xi}^{\mathrm{d}}$ and Yuqian Hao ${ }^{\mathrm{e}}$ \\ Hebei University of Science and Technology, Shijiazhuang 050000, China; \\ a183186143@qq.com,,b306806880@qq.com, chxaun@hebust.edu.cn, ${ }^{d} 1145362182 @ q q . c o m$,
}

e291850233@qq.com

Keywords: Hybrid AC/DC microgrid, Bidirectional AC/DC converter, Droop control.

\begin{abstract}
The hybrid AC/DC microgrid can combine the advantages of AC microgrid and DC microgrid, and it can reduce the loss caused by multi-stage power conversion, so it has become a hotspot in recent years. And the interlinking converter (ILC) between the AC subgrid and DC subgrid can ensure the balance of power of both sides and maintain the stability of the entire system. In this paper, the basic structure of hybrid AC/DC microgrid is introduced, the principle of traditional droop control is analyzed briefly. The main content of this paper is to analysis and summary the improved droop control methods of interlinking converter in hybrid AC/DC microgrid.
\end{abstract}

\section{Introduction}

Three phase AC power systems have existed for over 100 years due to their efficient transformation of AC power at different voltage levels and over long distance as well as the inherent characteristic from fossil energy driven rotating machines, so it have dominated electric power supply in the world for over hundreds years[1]. Recently more renewable power conversion systems are connected in low voltage AC distribution systems as distributed generators or AC microgrids due to environmental issues caused by conventional fossil fueled power plants[2]. However, DC power from photovoltaic (PV) panels or fuel cells has to be converted into ac using DC/DC boosters and DC/AC inverters inorder to connect to an ac grid. In an ac grid, embedded AC/DC and DC/DC converters are required for various home and office facilities to supply different dc voltages[3]. Recently, dc grids are resurging due to the development and deployment of renewable dc power sources and their inherent advantage for dc loads in commercial, industrial and residential applications. However, the DC microgrid can not replace the existing AC microgrid[4]. Thus, it is more likely that the architecture will be coexisted by the AC and DC subgrids, and connected by the power electronic control power converter, as shown in Fig.1.

In the hybrid AC/DC microgrid droop control can be used in DC bus, AC bus and distributed generation(DG), energy storage (SE). In the AC bus drooping control method, the AC bus voltage and frequency are controlled, and the demand power is shared between the input power sources. Compared with the AC bus droop control, the DC bus droop control is simpler because only the active power and voltage amplitude are related to the droop characteristics. Similarly, for proportional power sharing to DG rated power, the droop coefficient should be adjusted in inverse proportion to the rated power of DG or SE[5].Currently used in the microgrid converter control methods are mainly v/f droop control and PQ control[6].

\section{The Typical Hybrid AC/DC Microgrid}

The AC/DC hybrid microgrid contains DC bus and AC bus, it can reduce the power electronics conversion device and the losses generated by the conversion [7]. A typical AC/DC hybrid microgrid structure as the Fig. 1 shows. The DC and AC distribution networks are connected together by a three-phase AC/DC converter and their respective independent converters. AC/DC (or main) converters and their isolation transformers. DC power generators such as photovoltaic (PV) panels and 
fuel cell (FC) stacks are connected to DC networks through DC/DC boost converters. DC loads such as electric vehicles and speed control motors are tied to DC grid through DC/DC buck converters. DC energy storages such as batteries and super capacitors are connected to DC grid through bidirectional DC/DC converters. The three phase AC network of the hybrid grid, which can also be the existing low voltage distribution network, is connected to the utility grid through a transformer. AC power generators such as wind turbine generators and small diesel generators are connected to AC network through transformers. AC energy storages such as flywheels are connected to AC grid through $\mathrm{AC} / \mathrm{DC} / \mathrm{AC}$ converters and transformers. AC loads such as AC motors are connected to AC networks [8].

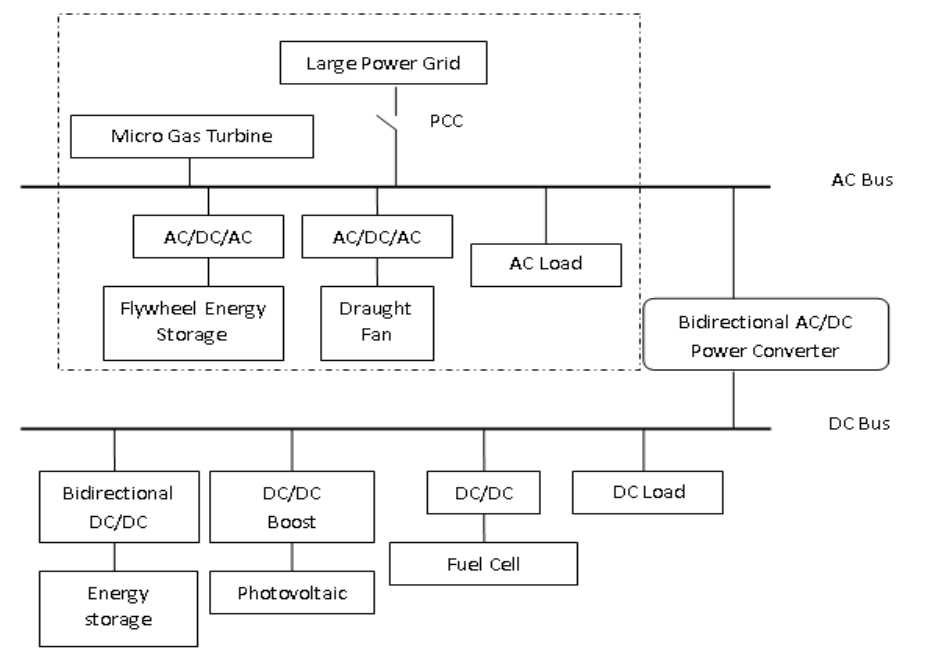

Fig. 1 hybrid AC/DC microgrid structure

\section{Traditional Droop Control Strategy in Microgrid}

When the microgrid in the peer-to-peer control mode, many distributed power supply using droop control methods. This control method is based on the correlation between the output active power and system frequency, reactive power and the terminal voltage of generator. When the system frequency is reduced, the generator's active power output will increased. When the terminal voltage is reduced and the reactive power of the generator output will be increased [9]. This control method controls the active and reactive power of the micro-source output separately, there is no need for communication between the units. And the micro-sources can achieve plug and play by using the method. It can also guarantee the balance of power and the unity of frequency when the microgrid in the island mode, with the characteristics of simple and reliable. $P / f, Q / U$ droop characteristic curve is shown in Fig. 2.
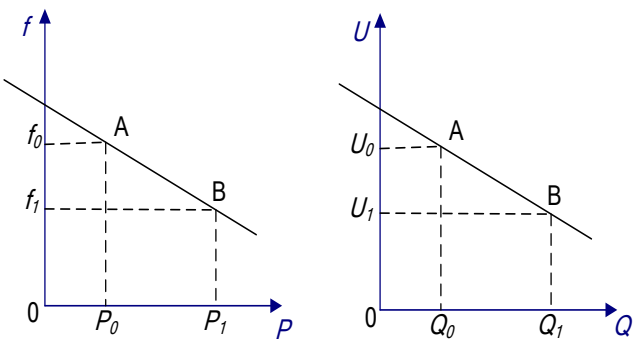

Fig. $2 \mathrm{P} / \mathrm{f}$ and $\mathrm{Q} / \mathrm{Udroop}$ characteristic curves

The control principle as shown in Figure 2, the figure shows the initial operating point of the distributed power system is A, the output active power is $P_{0}$, reactive power is $Q_{0}$, the system frequency is $f_{0}$, the voltage at the $\mathrm{AC}$ bus connected to the distributed power supply is $U_{0}$. When the system active load suddenly increases, the active power is insufficient, resulting in decreased frequency; system reactive load suddenly increases, the reactive power is insufficient, resulting in voltage amplitude decreased. Vice versa. When the system active load suddenly increase, the adjustment function of the droop control system is: When the frequency is reduced, the control system adjusts the active output power of the distributed power supply system to increase correspondingly 
according to the droop characteristic. At the same time, the load power is also reduced due to the frequency drop, and ultimately achieve the new power balance under the combined effect of the control system droop characteristics and load itself, that is, transition to operate on point B[10]. Droop relationship is:

$$
\begin{aligned}
& \left\{\begin{array}{l}
P=P_{0}+\left(f_{0}-f\right) K_{f} \\
Q=Q_{0}+\left(U_{0}-U\right) K_{U}
\end{array}\right. \\
& \left\{\begin{array}{l}
f=f_{0}+\left(P_{0}-P\right) K_{P} \\
U=U_{0}+\left(Q_{0}-Q\right) K_{Q}
\end{array}\right.
\end{aligned}
$$

In the peer mode, when the microgrid is in the island mode, each distributed power supply with the Droop control strategy in the microgrid is involved in the regulation of the voltage and frequency of the microgrid. In the case of a load change, the control strategy automatically divides the load change according to the droop coefficient[11]. That is, the distributed power supply by adjusting the frequency and amplitude of their respective output voltage, so that the micro-grid to a new steady-state operating point, and ultimately achieve a reasonable distribution of output power. In the grid-connected mode, the droop control strategy of the distributed power supply in the microgrid may not change, and the system operation mode is easy to switch seamlessly.

\section{Improved Droop Control of Interface Converter in Hybrid Microgrid}

\subsection{Piecewise Droop Control Method}

With the traditional droop control method may cause the converter to switch frequently. For example, when designing a control strategy for bidirectional AC/DC interface converters between AC and DC subgrids in hybrid microgrids, if the conventional droop control method is used, the deviation of the AC side output frequency or the DC side bus deviation occurs will make the converter action, will increase the loss of the converter, reducing the converter life.

The piecewise improved droop control method increase a idle mode, it can avoid frequent changes in the converter. And in the idle mode can be coordinated with the energy storage device control, increase the use of energy storage devices in hybrid microgrid[12]. The following description is take the piecewise improved droop characteristics of the bidirectional AC/DC power converter as an example. The piecewise improved droop characteristics of the bidirectional AC/DC power converter as shown in Fig.3.

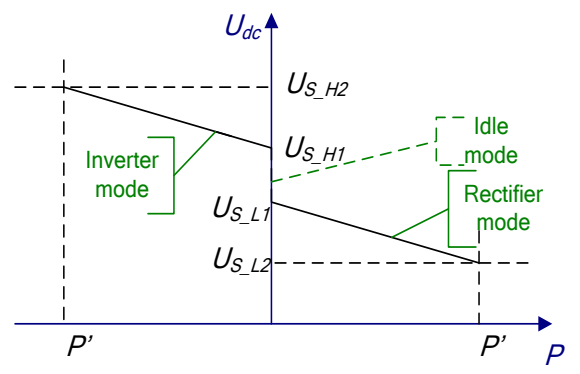

Fig. 3 Piecewise improved droop characteristic curves

In the figure $U_{S_{-} H I}$ and $U_{S_{-} L l}$ are the control threshold voltage of the bidirectional power converter. $U_{S_{-} H 2}$ and $U_{S_{-} L 2}$ are the adjustable DC bus voltage range bounds of the bidirectional AC/DC power converter according to the droop characteristics. The main reason for this is the rated output power of the bidirectional power converter. When the DC bus voltage fluctuates more than $U_{S_{-} H 2}$ and $U_{S_{-} L 2}$, the bidirectional AC/DC power converter can only transmit power at its rated power, and the bidirectional power converter does not have the effect of adjusting the DC bus voltage to a steady value. The improved droop characteristics are shown in Equation 3: 


$$
P_{d c}^{*}=\left\{\begin{array}{l}
-P^{\prime} \frac{U_{d c}-U_{S_{-} H 1}}{U_{S_{-} H 2}-U_{S_{-} H 1}}, U_{S_{-} H 1} \leq U_{d c}<U_{S_{-} H 2} \\
0, \quad U_{S_{-} L 1}<U_{d c}<U_{S_{-} H 1} \\
P^{\prime} \frac{U_{d c}-U_{S_{-} L 1}}{U_{S_{-} L 2}-U_{S_{-} L 1}}, U_{S_{-} L 2}<U_{d c} \leq U_{S_{-} L 1}
\end{array}\right.
$$

Where $P^{\prime}$ is the rated power of the bidirectional power converter; $P^{*} d c$ is the DC side transmission power reference value of the bidirectional power converter.

\subsection{Standardization Method}

That is, the DC side active power-voltage drop characteristics and AC side active power-frequency droop characteristics through the formula (4) for standardization, so that they can be placed in a coordinate system for analysis.

$$
\left\{\begin{array}{l}
f_{p u}=\frac{f-0.5\left(f_{\max }+f_{\min }\right)}{0.5\left(f_{\max }-f_{\min }\right)} \\
U_{p u}=\frac{U-0.5\left(U_{\max }+U_{\min }\right)}{0.5\left(U_{\max }-U_{\min }\right)}
\end{array}\right.
$$

Where $f_{p u}$ is the unitary value of the frequency; $f_{\max }, f_{\min }$ are the system frequency maximum and minimum; $U_{p u}$ is the voltage per unit value; $U_{\max }$ and $U_{\min }$ are the maximum and minimum values of the system voltage respectively. After the dispose of standardization, the frequency and voltage are limited to [-1,1], so that the droop control applied to the AC-DC microgrid is applied to the ILC of the AC/DC hybrid microgrid, as long as the $f_{p u}=U_{p u}, \mathrm{AC}$ and DC side power can be balanced. When $f_{p u} \neq U_{p u}$, the difference between after the dispose of PI controller to generate the active power reference on the ILC.

The proposed bidirectional AC/DC power converter control strategy between AC and DC hybrid microgrids in literature [13]adopts only one set of control strategies in grid-connected and isolated island mode, which is used in this method. The characteristics of DC bus voltage-active power and AC bus output frequency-active power should be unitized and put them in the same coordinate system. And then the difference between the values obtained on both sides of the AC and DC are normalized and through PI control, the improved control strategy can be obtained and it applies to both modes[3].

The literature[14]integrated the above two methods, first standardize the frequency of AC bus and the voltage of DC bus, and then placed in the same coordinate system, the difference between the two values through PI control to eliminate the steady-state error and the exchanging between the AC and DC microgrid power is calculated through related calculation. And then set the error action threshold, so that the operating range can be piecewise, so as to avoid frequent action.

\subsection{Power Control Method}

Different from the method of standardization, the method of power control no longer normalizes the acquisition signals on both sides of the AC/DC subgrid, but directly obtain the transmission power reference value according to the droop characteristics on both sides. And then compare the power reference values on both sides to determine the power flow, the difference as a reference value of the transmission power. This method is used in the literature[15]. When the frequency and voltage on the AC side are consistent with the large power grid, the control power flow can only consider the DC side. After simplified, the power values of AC side in the d-q coordinate system are $P=1.5 e_{d} i_{d}$, so you can control the flow of active power by current, and $i_{d}$ can be calculated by $u_{d c}$. The schematic diagram of the power quantity processing in the island mode is shown in Fig 4.

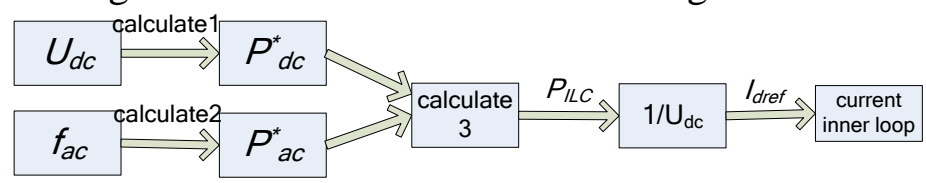

Fig. 4 Power control method illustration 


\subsection{Bidirectional Droop Control}

The bidirectional droop control method for the converter in an AC/DC hybrid microgrid is proposed by China Electric Power Research Institute in 2015, and it has applied for a patent. The method is based on the droop characteristics of $f-P$ and $U_{d c}-P$ on both sides of $\mathrm{AC}$ and $\mathrm{DC}$, and the active power flowing through the interface converter is controlled by AC bus frequency and DC bus voltage[11]. The power reference value of the converter is achieved from the output subtraction of two modules. Meanwhile, to reduce the deviation of voltage and frequency caused by droop control, a recovery control is designed to improve the power quality and the reliability of the hybrid microgrid. The bidirectional droop control can accurately coordinate the power transmission between AC subgrid and DC subgrid and make full use of the distributed energy. The transmission power is calculated according to the droop characteristics of the interlinking converter as shown in equation (5):

$$
P_{r e f}=P_{0}-K_{0}\left(U_{d c 0}-U_{d c}\right)+K_{f}\left(f_{0}-f\right)
$$

Where $P_{r e f}$ is the reference active power of the interlinking converter obtained from bidirectional droop control . $\mathrm{P}_{0}$ is the output active power rating of the interlinking converter, $f_{0}, U_{d c 0}$ are the AC bus frequency and DC voltage rating of the interlinking converter; $K_{f}, K_{0}$ are the droop coefficients of frequency and voltage, respectively. $f, U_{d c}$ are the actual value of AC bus frequency and DC bus voltage, respectively. The power and current dual loop control allows the converter's output power to track the reference power.

The bidirectional droop control may cause the DC bus voltage or the frequency of the AC bus to fall. To this end, the recovery control structure is proposed. After multiplying the value obtained by the PI control and the dead zone control, the compensation value of the active power is obtained to maintain the DC voltage and the AC frequency, improve power quality.

\subsection{Unify the Droop Characteristics of Both Sides with Euler Method}

Using the forward Euler approximation method to unify the droop curve characteristics of both sides, this method in some literatures also known as the new "net-net" droop control. The method mentioned in[16]is to unify the voltage-active power droop curves on both sides of the AC and DC, and exchange the power difference between the two sides by charging and discharging the DC side capacitor. The new droop characteristic is shown in equation (6).

$$
u_{a c, n}-u_{a c}=k_{H}\left(u_{d c, n}^{2}-u_{d c}^{2}\right)
$$

Where $u_{a c, n}$ is the AC bus voltage rating, $u_{a c}$ is the actual value of the AC bus, $k_{H}$ is the coefficient associated with the DC side capacitor, $u_{d c, n}$ and $u_{d c}$ are the DC bus voltage rating and the actual value, respectively.

The control strategy proposed in[17]is to unify the droop characteristics of AC side frequency-active power and DC side voltage-active power by Euler method, and get the new droop characteristic formula as shown in equation (7).

$$
\left(f_{0}-f\right)=k\left(U_{d c 0}^{2}-U_{d c}^{2}\right)
$$

Where $f_{0}$ is the AC side reference frequency; $U_{d c 0}$ is the DC reference voltage; $k$ is the coefficient related to the DC side capacitance; $f, U_{d c}$ is the measured data.

The deadband region of the DC side voltage and the both sides frequency is set in this document. When the DC side voltage and the AC side frequency fluctuate in the dead zone, the interlinking converter does not operate, the power balance is solved internally by the microgrid. When the dead zone is exceeded, the new AC/DC droop control is adopted, and the power balance of the microgrid at both sides is realized by the improved two-stage droop control.

\section{Conclusion}

This paper firstly pointed out that the hybrid AC/DC microgrid has a good prospect and significance, and then introduces a typical structure of the hybrid AC/DC microgrid briefly. Traditional droop control strategy applied in the microgrid is also just analyzed briefly. The main points of this paper are the summary and analysis of the improved droop control methods, including 
piecewise droop control, standardization, power control, bidirectional droop control and unify the droop characteristics of both sides with Euler method .These methods improved the traditional droop method, and they are more suitable for hybrid AC/DC microgrid interlinking converter, so that the interlinking converter can maintain the balance of $\mathrm{AC}$ and DC power and ensure the stable operation of the entire microgrid .

\section{Acknowledgments}

Supported by the Planned Science and Technology Project of Hebei Province, China (No.16214510D)

\section{References}

[1]. F. Nejabatkhah,Yun Wei Li.Overview of Power Management Strategies of Hybrid AC/DC Microgrid.IEEE Transactions on Power Electronics.Vol.30(2015) No.12,p.7072-7089

[2]. Xiong Liu,Peng Wang,Poh Chiang Loh.A Hybrid AC/DC Microgrid and Its Coordination Control.IEEE Transactions on Smart Grid.Vol.2(2011) No.2,p.278-286

[3]. Poh Chiang Loh,Ding Li,Yi Kang Chai.et al.Autonomous Control of Interlinking Converter With Energy Storage in Hybrid AC-DC Microgrid.Industry Applications IEEE Transactions on.Vol.49(2013) No.3,p.1374-1382

[4]. Poh Chiang Loh,Ding Li, Yi Kang Chai.et al.Autonomous Operation of Hybrid Microgrid With AC and DC Subgrids.IEEE Transactions on Power Electronics.Vol.28(2013) No.5,p.2214-2223

[5]. Xiao Sheng Wang,Kai Sun, Yun Wei Li.Review of Control Strategies for Bidirectional Interfacing Review of Control Strategies for Bidirectional Interfacing

Review of Control Strategies for Bidirectional Interfacing Converters in Hybrid AC/DC Microgrid.Journal of Power Supply.Vol.14 (2016) No.2, p.70-79

[6]. Cheng Shan Wang.Microgrid Analysis and Simulation Theory.Science Press,2013年, p.

[7]. Yong Qiang Zhu,Li Hu Jia,Bing Qian Cai.et al.Overview on Topologies and Basic Control Strategies for Hybrid AC/DC Microgrid.High Voltage Engineering.Vol.42(2016) No.9,p.2756-2767

[8]. .Peng Wang,Xiong Liu,Chi Jin.et al.A hybrid AC/DC micro-grid architecture, operation and control.2011) No.1-8

[9]. Yi Wei Zhang,Jian Guang Chu.Research on Hybrid Microgrid Three-phase Converter. Manager' Journey.Vol.2015) No.8Z,p.

[10]. Hui Peng Li,Xiao Qing Han, Yu Yang.et al.Control Method of Hybrid Microgrid Three-phase Converter.Journal of Henan University of Science and Technology: Natural Science.Vol.2014) No.2,p.55-61

[11]. .Ze Gao,Jian Hua Yang, Yu Ji.et al.Bidirectional Droop Control of AC/DC Hybrid Microgrid Interlinking Converter.Southern Power System Technology.Vol.9(2015) No.5,p.82-87

[12]. Jia Yi Liu,Wen Ping Qin,Xiao Qing Han.et al. Control Method of Interlink-Converter in DC Microgrid.Power System Technology.Vol.38(2014) No.2,p.304-310

[13]. .Jia Yi Liu:Research on the Control Method of Interlink-converter in Hybrid Microgrid(Taiyuan University of Technology,2014).p.

[14]. .Wen Chao Xie, Yong Qiang Zhu,Shao Fei Du.et al. State Key Laboratory of Alternate Electrical Power System with $\mathrm{R}$ enewable Energy Sources.Electric Power Construction.Vol.37(2016) No.10,p.9-15

[15]. Lei Tang,Cheng Bi Zeng,Hong Miao.et al.One novel control strategy of the AC / DC bi-directional power converter in micro-grid.Power System Protection and Control.Vol.2013) No.14,p.13-18

[16]. Li Hu Jia,Yong Qiang Zhu,Shao Fei Du.et al.Control Method of AC/DC Hybrid Microgrid Interlinking Converter.Automation of Electric Power Systems.Vol.40(2016) No.24,p.98-104 
[17]. Navid Eghtedarpour,Ebrahim Farjah.Power Control and Management in a Hybrid AC/DC Microgrid.IEEE Transactions on Smart Grid.Vol.5(2014) No.3,p.1494-1505 